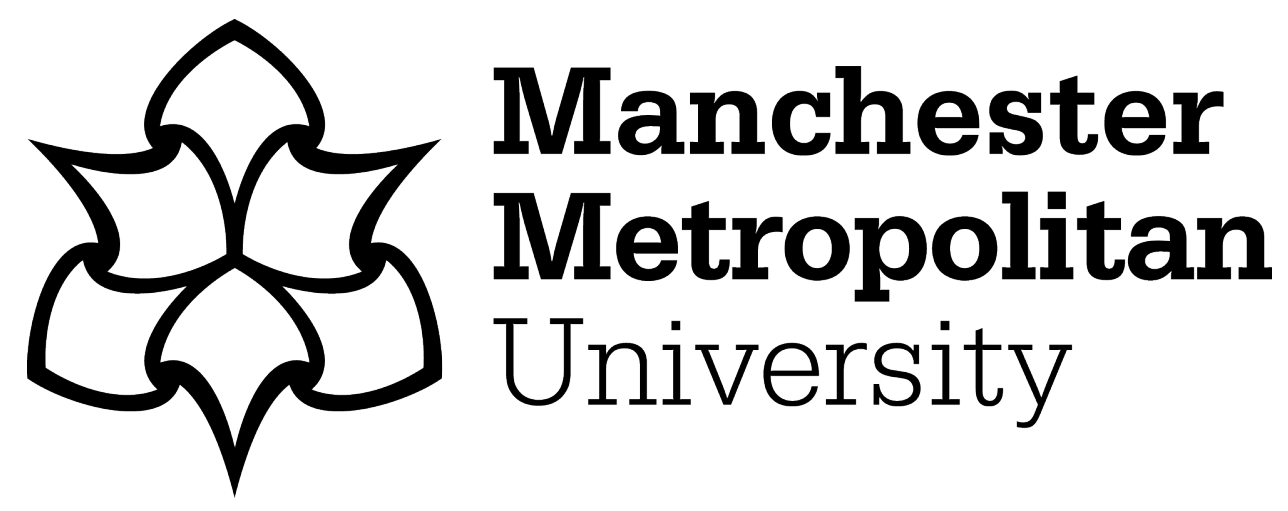

Jarvis, HL, Brown, SJ, Butterworth, C, Jackson, K, Clayton, A, Walker, L, Rees, N, Price, M, Groenevelt, R and Reeves, ND (2021) The gait profile score characterises walking performance impairments in young stroke survivors. Gait and Posture, 91. pp. 229-234. ISSN 0966-6362

Downloaded from: https://e-space.mmu.ac.uk/628725/

Version: Accepted Version

Publisher: Elsevier

DOI: https://doi.org/10.1016/j.gaitpost.2021.10.037

Usage rights: Creative Commons: Attribution-Noncommercial-No Derivative Works 4.0

Please cite the published version 


\title{
The gait profile score characterises walking performance impairments in young stroke survivors
}

\author{
Hannah L. Jarvis ${ }^{a, *}$, Steven J. Brown ${ }^{\text {a }}$, Claire Butterworth ${ }^{\mathrm{b}}$, Karl Jackson ${ }^{\mathrm{c}}$, Abigail Clayton ${ }^{\mathrm{d}}$, \\ Louisa Walker $^{\mathrm{e}}$, Nia Rees ${ }^{\mathrm{e}}$, Michelle Price ${ }^{\mathrm{f}}$, Renee Groenevelt $^{\mathrm{g}}$, Neil D. Reeves ${ }^{\mathrm{a}}$ \\ ${ }^{a}$ Research Centre for Musculoskeletal Science and Sports Medicine, Department of Life Sciences, Faculty of Science and Engineering, Manchester Metropolitan University, \\ Manchester, UK \\ ${ }^{\mathrm{b}}$ Cardiff and Vale University Health Board, Physiotherapy, Llandough Hospital, Penlan Road, Cardiff CF64 2XX, UK \\ ${ }^{\mathrm{c}}$ Betsi Cadwaladr University Health Board, Physiotherapy, Ysbyty Gwynedd, Bangor LL57 2PW, UK \\ ${ }^{\mathrm{d}}$ Swansea Bay University Health Board, Therapy Services, Heol Maes Eglwys, Swansea SA6 6NL, UK \\ ${ }^{\text {e }}$ Cwm Taf Bro Morgannwg University Health Board, Physiotherapy Department, Keir Hardie Health Park, Merthyr Tydfil CF48 1BZ, UK \\ ${ }^{\mathrm{f}}$ Powys Teaching Health Board, Neuro Rehabilitation, Newtown Hospital, Newtown SY6 2DW, UK \\ ${ }^{\mathrm{g}}$ Hywel Dda University Health Board, Physiotherapy, Fishguard Road, Haverfordwest, Pembrokeshire SA61 2PZ, UK
}

\section{A R T I C L E I N F O}

\section{Keywords:}

Stroke

Young stroke survivor

Gait Profile Score

GPS

Gait

Walking speed

\begin{abstract}
A B S T R A C T
Background: The Gait Profile Score (GPS) provides a composite measure of the quality of joint movement during walking, but the relationship between this measure and metabolic cost, temporal (e.g. walking speed) and spatial (e.g. stride length) parameters in stroke survivors has not been reported.

Research Question: The aims of this study were to compare the GPS (paretic, non-paretic, and overall score) of young stroke survivors to the healthy able-bodied control and determine the relationship between the GPS and metabolic cost, temporal (walking speed, stance time asymmetry) and spatial (stride length, stride width, step length asymmetry) parameters in young stroke survivors to understand whether the quality of walking affects walking performance in stroke survivors.

Methods: Thirty-nine young stroke survivors aged between 18 and 65years and 15 healthy age-matched ablebodied controls were recruited from six hospital sites in Wales, UK. Joint range of motion at the pelvis, hip, knee and ankle, and temporal and spatial parameters were measured during walking on level ground at self-selected speed with calculation of the Gait Variable Score and then the GPS.

Results: GPS for the paretic leg $\left(9.40^{\circ}(8.60-10.21) \mathrm{p}<0.001\right)$, non-paretic leg $\left(11.42^{\circ}(10.20-12.63) \mathrm{p}<0.001\right)$ and overall score $\left.\left(11.18^{\circ}(10.26-12.09) \mathrm{p}<0.001\right)\right)$ for stroke survivors were significantly higher than the control $\left(4.25^{\circ}(3.40-5.10), 5.92^{\circ}(5.11(6.73))\right.$. All parameters with the exception of step length symmetry ratio correlated moderate to highly with the GPS for the paretic, non-paretic, and/or overall score $(\rho=<-0.732$ (p $<$ 0.001)).

Significance: The quality of joint movement during walking measured via the GPS is directly related to the speed and efficiency of walking, temporal (stance time symmetry) and spatial (stride length, stride width) parameters in young stroke survivors.
\end{abstract}

\section{Introduction}

Adults who have had a stroke walk slower and less efficiently with distinct differences and/or asymmetry in temporal (e.g., stance time), spatial (e.g., stride length, step length), joint kinematic and kinetic parameters of the paretic and non-paretic limbs when compared to healthy able-bodied controls [1-12]. Muscle weakness and/or spasticity, impaired balance, and increased reluctance to bear weight on the paretic limb can cause abnormally increased or decreased joint range of motion, reduced joint moments and decreased power absorption and/or generation by muscles groups of the lower limb (e.g., calf muscles) [3,8-12].

Whilst gait kinematics and kinetics provide in-depth detail regarding the biomechanical function of the lower limb during walking, interpreting this data clinically can be challenging [13]. It also generates vast

\footnotetext{
* Corresponding author.

E-mail address: Hannah.Jarvis@mmu.ac.uk (H.L. Jarvis).
} 
amount of data and parameters which are important to assess [14], but identifying which and how these parameters are related to walking performance parameters such as metabolic cost and walking speed is difficult.

In response to this, several researchers have developed gait assessment measures that provide an overall and composite representation of gait kinematics and the quality of walking which can be easily interpreted in a clinical setting. These include the Gait Deviation Index [15], Gillete Gait Index [16] and the Gait Profile Score (GPS) [13].

The GPS has gained considerable popularity and has been widely reported in a variety of clinical populations due to its simplicity and good reliability $[13,17-20]$. It is derived by calculating the root mean square difference between the angular trajectories of a participant compared to a reference healthy able-bodied sample with no pathology [13]. The GPS provides an indication of the quality of movement and because it is measured in degrees it offers a more direct clinical interpretation for what the score outcome means (i.e., enabling a direct comparison to a healthy-able-bodied individual) $[13,18]$. A GPS of between $5^{\circ}$ and $6^{\circ}$ has been routinely reported for healthy able-bodied control population $[13,18,21,22]$, therefore inferring scores above this indicate poor quality of walking or an abnormal gait pattern.

Only three studies have reported the GPS in stroke survivors [19,20, 22]. Fukuchi et al. [22] used the GPS to develop a specific GPS sensitive to walking speed and Devetak et al. [19] tested its intra and inter-session reliability. Bigoni et al. [20] reported that the GPS was fairly correlated to clinical measures such as the 10-metre walk test, functional ambulation category and a good correlation to the Berg Balance Scale. No study has investigated the relationship between the GPS and the efficiency of walking, temporal, and spatial parameters, and yet these are commonly altered post-stroke which we can hypothesise are caused by abnormal joint kinematics.

There are relatively few gait studies in young stroke survivors, and none have measured the GPS in young stroke survivors. Understanding how the quality and quantity of walking is affected post-stroke and its relationship to walking speed and metabolic cost may help better inform rehabilitation programmes for young stroke survivors [1]. As young stroke survivors are less likely to be affected by age-related changes associated with bone degeneration and muscle weakness, capturing the GPS in young stroke survivors may help to determine the true effect of a stroke on this gait measure without the confounding factor of age.

The aims of this study were to: 1 . Compare the GPS (paretic, nonparetic and overall score) of young stroke survivors to the GPS of the healthy able-bodied control, and 2. Determine the relationship between the GPS scores and metabolic cost, temporal (walking speed, stance time asymmetry) and spatial (stride length, stride width, step length asymmetry) parameters in young stroke survivors. We hypothesise that young stroke survivors will walk with a higher GPS than the control and that the higher the GPS the slower the walking speed and less efficient young stroke survivors are at walking, suggesting a strong relationship between these variables.

\section{Methods}

This study was approved by the NHS Research Ethics Committee (Wales Regional Ethics Committee 618/WA/0265) and Health Research Authority (UK) and Manchester Metropolitan University Research Ethics Committee. Informed written consent to take part in this study was obtained from each participant and thirty-nine individuals aged between 18 and 65 years were recruited and agreed to participate from six health boards in Wales, UK between September 2018 and October 2018. Inclusion criteria for stroke participants were 18-65 years of age, haemorrhage or infarct stroke within the last $\mathrm{t} 3$-years that is evident from a computerised tomography (CT) scan, singular lesion that occurred in one hemisphere in the brain and able to walk continuously for at least three minutes. Stroke participants with multiple lesions in different hemispheres in the brain or a lesion in the brainstem were excluded.
Participants who had a stroke but were also diagnosed with a disease that was the predominant health concern that limited their ability to walk, were also excluded from this study.

Fifteen control participants were recruited and agreed to participate. Inclusion criteria was no history of stroke, neurological, musculoskeletal, cardiovascular, auto immune, or respiratory disease. Very physically active (e.g., elite athletes) individuals, or participants who smoke or have smoked in the past were excluded from this study.

\subsection{Outcome measures}

\subsubsection{Demographic data}

Demographic data included age, body mass, height, time since stroke, type of stroke, and whether the right or left side was predominantly affected by the stroke was used to determine the paretic and nonparetic limb. A summary of demographic measures are presented in the $s$ manuscript, individual and other demographic measures are available in Supplementary Data Table 3 and here [1].

\section{Methods}

\subsection{Joint kinematics}

An eight-camera optoelectronic motion capture system (Oqus, Qualisys motion capture system, Qualisys, Sweden) was placed around a $15 \mathrm{~m}$ walkway with four Kistler force plates embedded within a walkway to capture three-dimensional walking biomechanics. Retroreflective markers were placed on anatomical landmarks to define joint centres and body segments using the marker set previously described in previous stroke research [23]. Please see Table 1 (Supplementary data). Motion analysis data was collected at $120 \mathrm{~Hz}$ and ground reaction forces at $1200 \mathrm{~Hz}$. A static standing trial was recorded for each participant to calculate a participant-specific calculation of the location of joint centres and then participants completed seven walking trials of approximately $5 \mathrm{~m}$ in length. The average of these walking trials was then used per joint to calculate the GPS.

Data were digitised in Qualisys Track Manager, Qualisys, Sweden and then exported for modelling and analysis within Visual 3D (C-Motion, Rochelle, USA). A model specific to the height and body mass of each participant was created. The inertial parameters were calculated for the pelvis, hip, knee, ankle, and foot using inverse dynamics. This allows specific constraints to be applied at the joints of the virtual model so to limit rotation and or translation. The pelvis permitted six degrees of freedom, but only sagittal, coronal, and transverse plane rotation was permitted at all other joints. Gait events (initial contact and foot off) were defined from contact with the force plates. Visual 3D was used to calculate and extract wave form angles for the pelvis, hip, knee, ankle, and foot for each plane of motion.

\subsection{Calculation of GPS}

The GPS [13] was calculated from the gait variable score which is calculated by the root mean square difference between joint angles of each participant and the average of the healthy able-bodied participants during the gait cycle. There are fifteen gait variable scores. Four from the sagittal plane (pelvis anterior/posterior tilt, hip flexion/extension, knee flexion/extension, ankle dorsiflexion/plantarflexion), two from the coronal plane (pelvis up/down, hip adduction/abduction) and three from the transverse plane (pelvis internal/external, hip internal/external and foot progression) for each leg and only pelvis kinematics from the paretic/left leg were used.

\subsection{Temporal and spatial parameters}

Gait events (initial contact and foot off) were defined from contact with force plates and used to calculate temporal and spatial parameters. 
Table 1

Presents mean and 95\% confidence interval for participant demographics. n/a: not applicable.

\begin{tabular}{lll}
\hline & Stroke $(\mathbf{n}=\mathbf{3 9})$ & Control $(\mathbf{n}=\mathbf{1 5})$ \\
\hline Age (years) & $49.60(45.98-53.22)$ & $44.53(36.17-52.88)$ \\
Male/Female & $\mathrm{n}=32 / \mathrm{n}=7$ & $\mathrm{n}=7 / \mathrm{n}=8$ \\
Body mass (kg) & $93.25(84.86-101.64)$ & $74.98(67.78-82.18)$ \\
Height (m) & $1.71(1.65-1.77)$ & $1.73(1.69-1.78)$ \\
Time since stroke (months) & $12.86(9.17-16.56)$ & $\mathrm{n} / \mathrm{a}$ \\
\hline
\end{tabular}

\section{Table 2}

Presents mean and 95\% confidence interval for participant demographics, walking speed, metabolic cost, GPS (paretic, non-paretic limbs and overall score), stride length, stride width, step length asymmetry and stance time asymmetry for young stroke survivors compared to controls. Left leg was used for control participants to compare to GPS paretic, non-paretic and overall score.

\begin{tabular}{lll}
\hline & Stroke $(\mathbf{n}=39)$ & Control $(\mathbf{n}=\mathbf{1 5})$ \\
\hline Walking Speed $(\mathrm{m} / \mathrm{s})$ & $0.81(0.69-0.93)$ & $1.42(1.33-1.51)$ \\
$p$ & $<0.001$ & \\
Metabolic Cost $(\mathrm{ml} / \mathrm{kg} / \mathrm{m})$ & $0.29(0.24-0.34)$ & $0.15(0.14-0.15)$ \\
$p$ & $<0.001$ & \\
GPS Paretic leg $\left(^{\circ}\right)($ Left leg control) & $9.40(8.60-10.21)$ & $4.25(3.40-5.10)$ \\
$p$ & 0.001 & \\
GPS Non-paretic leg $\left(^{\circ}\right)$ (Left leg & 11.42 & $4.25(3.40-5.10)$ \\
$\quad$ control) & $(10.20-12.63)$ & \\
$p$ & $<0.001$ & \\
GPS Overall $\left(^{\circ}\right)$ & 11.18 & $5.92(5.11-6.73)$ \\
& $(10.26-12.09)$ & \\
$p$ & 0.014 & \\
Stride Length (m) & $0.89(0.77-1.00)$ & $1.33(1.24-1.41)$ \\
$P$ & $<0.001$ & \\
Stride Width (m) & $0.26(0.18-0.34)$ & $0.12(0.11-0.14)$ \\
$p$ & $<0.001$ & \\
Step Length asymmetry & $1.25(0.89-1.62)$ & $0.93(0.87-0.98)$ \\
$p$ & $<0.001$ & \\
Stance time asymmetry & $0.95(0.91-0.99)$ & $1.01(0.96-1.05)$ \\
$p$ & $<0.001$ & \\
\hline
\end{tabular}

Temporal parameters included walking speed, stance time and spatial parameters included stride length, stride width, and step length. Symmetry ratios for step length and stance time were calculated using the recommended calculation by other studies $[1,4]$. See Supplementary Table 2 for description of temporal and spatial parameters calculation.

\subsection{Metabolic cost}

To capture metabolic energy expenditure during walking, participants were fitted with a gas analyser (Cortex Metalyser, Biophysik) which measures oxygen consumption. All participants walked at their self-selected speed for three minutes up and down a $15 \mathrm{~m}$ long runway with timing gates situated $5 \mathrm{~m}$ from either end of the runway to measure walking speed. The last minute of oxygen consumption data from each participant was used for analysis. Oxygen consumption underwent normalisation to body mass and to calculate the metabolic cost of walking, which is a measure of efficiency, oxygen consumption was divided by walking speed, which indicates the millilitres of oxygen uptake required per kilogram of bodyweight to cover a metre of ground.

\subsection{Statistical analysis}

Statistical analysis was conducted using SPSS Version 24. All data were checked for normality using the Shapiro-Wilko test and measures of skewness and kurtosis. Mean and 95\% confidence interval were calculated for each parameter. The GPS for the paretic, non-paretic and overall score, metabolic cost, temporal, and spatial parameters of young stroke survivors were compared to the healthy able-bodied control (left leg score was compared to paretic and non-paretic leg) using Mann
Table 3

presents the Gait Variable Score for the paretic, non-paretic and control limbs for each parameter used to calculate the Gait Profile Score. No formal statistical analysis was conducted to compare between groups as multiple comparisons would be required and the overall gait profile score should be used for formal between group analysis.

\begin{tabular}{|c|c|c|c|}
\hline \multirow[t]{2}{*}{ Parameter } & \multicolumn{2}{|l|}{ Stroke $(\mathrm{n}=39)$} & \multirow{2}{*}{$\begin{array}{l}\text { Control }(\mathrm{n}=15) \\
\text { Gait Variable } \\
\text { Score (left leg } \\
\text { only) }\end{array}$} \\
\hline & $\begin{array}{l}\text { Gait Variable } \\
\text { Score Paretic leg }\end{array}$ & $\begin{array}{l}\text { Gait Variable Score } \\
\text { Non-paretic leg }\end{array}$ & \\
\hline $\begin{array}{l}\text { Pelvis Ant/ } \\
\text { Post Tilt }\end{array}$ & $6.13(5.01-7.25)$ & $6.83(5.34-8.32)$ & $3.95(2.35-5.55)$ \\
\hline Hip Flex/Ext & $10.43(8.79-12.06)$ & $10.55(8.17-12.94)$ & $5.11(3.31-6.91)$ \\
\hline $\begin{array}{l}\text { Knee Flex/ } \\
\text { Ext }\end{array}$ & $\begin{array}{l}13.24 \\
(10.80-15.67)\end{array}$ & $20.37(17.13-23.60)$ & $4.33(3.49-5.17)$ \\
\hline $\begin{array}{l}\text { Ankle Dor/ } \\
\text { Pla }\end{array}$ & $9.14(8.02-10.26)$ & 11.15 (9.36-12.93) & $4.60(2.99-6.22)$ \\
\hline $\begin{array}{l}\text { Pelvis Up/ } \\
\text { Dn }\end{array}$ & $4.39(3.73-5.06)$ & $4.76(4.12-5.40)$ & $1.95(1.37-2.53)$ \\
\hline $\begin{array}{l}\text { Hip Add/ } \\
\text { Abd }\end{array}$ & $4.82(3.99-5.65)$ & $5.00(4.21-5.80)$ & $3.26(2.37-4.15)$ \\
\hline $\begin{array}{l}\text { Pelvic Int/ } \\
\text { Ext }\end{array}$ & $5.14(3.93-6.35)$ & $5.69(3.91-7.47)$ & $2.48(1.80-3.17)$ \\
\hline Hip Int/Ext & 8.97 (7.16-10.78) & $6.50(5.50-7.50)$ & $4.30(3.14-5.45)$ \\
\hline Foot Int/Ext & $10.13(8.19-12.06)$ & $12.27(9.37-15.18)$ & $4.31(2.86-5.75)$ \\
\hline GPS & $9.40(8.60-10.21)$ & $11.42(10.20-12.63)$ & $4.25(3.40-5.10)$ \\
\hline $\begin{array}{l}\text { GPS overall } \\
\text { score }\end{array}$ & $11.18(10.26-12.09)$ & & $5.92(5.11-6.73)$ \\
\hline
\end{tabular}

Whitney $U$ tests with a Bonferroni correction. The GPS of the paretic leg was compared to the non-paretic leg for young stroke survivors using a Mann Whitney $U$ test with Bonferroni correction. The GPS for the paretic, non-paretic and overall score was correlated to metabolic cost, temporal and spatial parameters using a Spearman's rho correlation.

\section{Results}

\subsection{Demographic data}

Young stroke survivors were thirty-two male and seven females, aged 49.60years (45.98-53.22)) and time since stroke 12.9 months (9.2-16.6)) (Table 1). All with confirmed stroke diagnosis by CT scan (n $=10$ haemorrhage, $\mathrm{n}=29$ ischaemic), the paretic limb was the right for $\mathrm{n}=20$ and left for $\mathrm{n}=19$ participants. Supplementary Data Table 3 provides demographic and neurological data for stroke participants. Control participants were seven male and eight females (Table 1).

4.1.1. Comparison of the GPS, temporal and spatial parameters and metabolic cost of young stroke survivors compared to the healthy able-bodied control

GPS for the paretic leg $\left(9.40^{\circ}(8.60-10.21) \mathrm{p}<0.001\right)$, non-paretic leg $\left(11.42^{\circ}(10.20-12.63) \mathrm{p}<0.001\right)$ and overall score $\left(11.18^{\circ}\right.$ $(10.26-12.09) \mathrm{p}<0.001)$ ) were significantly higher than the control $\left(4.25^{\circ}(3.40-5.10), 5.92^{\circ}\right.$ (5.11 (6.73)) (Table 2). The GPS of the paretic leg was significantly less than the than the non-paretic leg $(\mathrm{p}=0.010)$.

Young stroke survivors walked significantly slower and less efficiently $(0.81 \mathrm{~m} / \mathrm{s}(0.69-0.93) \mathrm{p}<0.001,0.29 \mathrm{ml} / \mathrm{kg} / \mathrm{m}(0.24-0.34) \mathrm{p}<$ $0.001)$, compared to the control (1.42 m/s (1.33-1.51), $0.15 \mathrm{ml} / \mathrm{kg} / \mathrm{m}$ $(0.14-0.15))$ (Table 2). Stride length $(0.89 \mathrm{~m}(0.77-1.00) \mathrm{p}=<0.001)$ and width $0.26 \mathrm{~m}((0.18-0.34) \mathrm{p}<0.001)$ were significantly shorter and wider for young stroke survivors compared to the control $(1.33 \mathrm{~m}$ (1.24-1.41), $0.12 \mathrm{~m}(0.11-0.14))$ (Table 1). Step length symmetry ratio was greater $(1.25(0.89-1.62) \mathrm{p}<0.001))$ and stance time symmetry ratio $(0.95(0.91-0.99) \mathrm{p}<0.001))$ less for young stroke survivors compared to the control (0.93 (0.87-0.98), 1.01 (0.96-1.05)) (Table 2). 

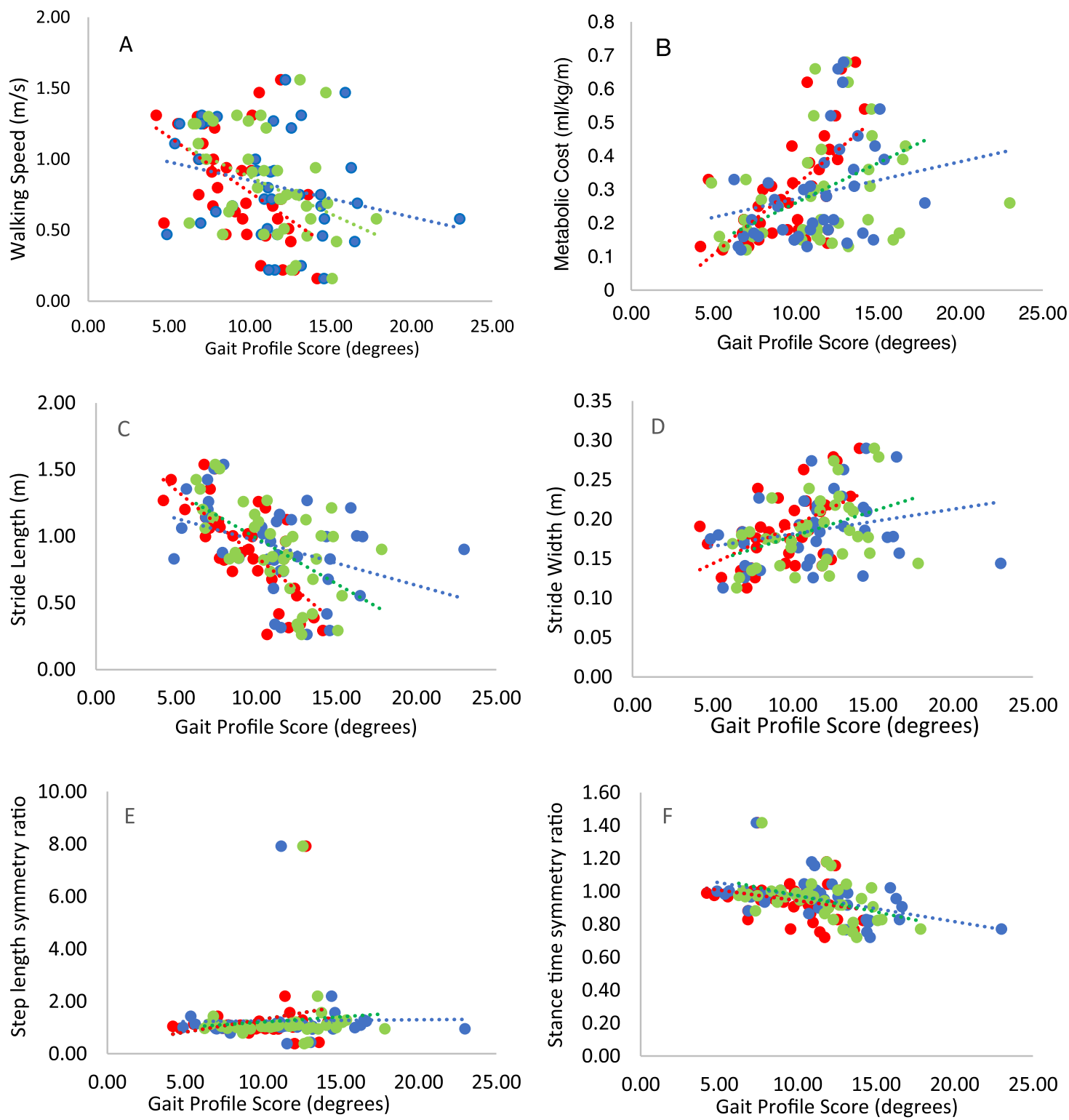

Fig. 1. Scatter plot of the GPS for the paretic leg (red dots), non-paretic leg (blue leg) and overall score (green dots) correlated to: A walking speed, B. metabolic cost, C. stride length, D. stride width, E. step length symmetry ratio, F. stance time symmetry ratio for young stroke survivors. Red dotted line represents trendline for paretic leg, blue dotted line represents trendline for non-paretic leg, green dotted line represents trendline for overall score.

\subsubsection{Relationship between the GPS, temporal and spatial parameters, and} metabolic cost in young stroke survivors

All gait parameters with the exception of step length symmetry ratio correlated moderate to highly to the GPS (Table 3, Fig. 1). Higher correlations were observed between the GPS of the paretic leg and the overall score than the non-paretic leg across all parameters. Walking speed correlated negatively and moderately to the GPS for the paretic leg $(\rho=-0.543(p<0.001)$ and overall score $(\rho=-0.397(p=0.012))$, whilst metabolic cost correlated positively and strongly to the GPS for the paretic $(\rho=0.644(\mathrm{p}<0.001))$ and overall score $(\rho=0.472(\mathrm{p}=$ 0.002) (Table 3, Fig. 1). Stride length correlated negatively and strongly to the GPS for the paretic $(\rho=-0.732(\mathrm{p}<0.001))$, moderately to the non-paretic $(\rho=-0.418(\mathrm{p}=0.008))$ and overall score $(\rho=-0.572(\mathrm{p}$ $<0.001)$ ). Similarly, stride width correlated with the GPS positively and moderately for the paretic leg $(\rho=0.543(\mathrm{p}=0.001)$, and the overall score $(\rho=0.408$ ( $p=0.01$ ) (Table 3 , Fig. 1$)$. Stance time symmetry ratio was negatively and moderately correlated, but only for the non-paretic leg $(\rho=-0.499(\mathrm{p}=0.001))$ and overall score $(\rho=-0.454(\mathrm{p}=$ 0.004)) (Table 3, Fig. 1). Table 4.

\section{Discussion}

This study reports for the first time a measure of the GPS in young stroke survivors. The GPS correlated against key gait variables and shows that a stroke not only affects the magnitude of joint movement (as previously reported $[8,12,24]$, but also the quality of movement measured via the GPS and is related to the speed and efficiency of walking, temporal (stance time symmetry) and spatial (stride length, stride width) parameters in young stroke survivors. It can be challenging to choose which individual parameters may be most useful to measure gait performance, but the GPS provides a composite measure of the quality of gait which correlates to other easily measured walking performance variables.

The GPS correlated moderately with nearly all gait parameters, except for step length symmetry ratio. Moderate and strong correlations were recorded between the GPS of the paretic leg and overall score with walking speed, metabolic cost, stride length and stride width. The results indicate that with a higher GPS, walking speed will decrease, metabolic cost increases, stride length shortens, and stride width widens. The 
Table 4

presents Spearman Rho correlation coefficients for Gait Profile Score (paretic, non-paretic limbs and overall score) correlated to walking speed, metabolic cost, stride length, stride width, step length asymmetry and stance time asymmetry for young stroke survivors.

\begin{tabular}{llll}
\hline Parameter & \multicolumn{2}{l}{ Stroke $(\mathrm{n}=39)$} & \\
\cline { 2 - 4 } & GPS Paretic & $\begin{array}{l}\text { GPS Non- } \\
\text { paretic }\end{array}$ & GPS Total \\
\hline Walking Speed $(\mathrm{m} / \mathrm{s})$ & $-0.543(\mathrm{p}<$ & $-0.241(\mathrm{p}=$ & $-0.397(\mathrm{p}=$ \\
& $0.001)$ & $0.139)$ & $0.012)$ \\
Metabolic Cost $(\mathrm{ml} / \mathrm{kg})$ & $0.644(\mathrm{p}=$ & $0.301(\mathrm{p}=0.06)$ & $0.472(\mathrm{p}=$ \\
m) & $<0.001)$ & & $0.002)$ \\
Stride Length $(\mathrm{m})$ & $-0.732(<0.001)$ & $-0.418(\mathrm{p}=$ & $-0.572(\mathrm{p}<$ \\
& & $0.008)$ & $0.001)$ \\
Stride Width $(\mathrm{m})$ & $0.543(\mathrm{p}=$ & $0.341(0.034)$ & $0.408(\mathrm{p}=$ \\
& $0.001)$ & & $0.01)$ \\
Step length symmetry & $0.06(\mathrm{p}=0.723)$ & $0.108(\mathrm{p} 0.514)$ & $0.128(\mathrm{p}=$ \\
ratio & & & $0.437)$ \\
Stance time symmetry & $-0.256(\mathrm{p}=$ & $-0.499(\mathrm{p}=$ & $-0.454(\mathrm{p}=$ \\
ratio & $0.115)$ & $0.001)$ & $0.004)$ \\
\hline
\end{tabular}

results also suggest that the quality of joint kinematics of the non-paretic limb is more affected than the paretic limb which was also reported by others [19]. We can hypothesise that this is due to compensation strategies by the non-paretic limb to accommodate for the reduced function of the paretic limb.

The negative correlation between stance time symmetry ratio and the GPS for the non-paretic leg and overall score further supports the notion that due to muscle weakness or spasticity, stroke survivors are reluctant to bear weight on to the paretic limb [1]. Therefore, rehabilitation strategies should focus on promoting weight acceptance by the paretic limb to improve the quantity and quality of gait post-stroke. This could include specific strengthening of the knee and hip extensors, knee flexors and ankle plantarflexors in a functionally relevant manner/situation to promote weight acceptance during gait.

This is the first time the GPS has been reported in young stroke survivors. Previous research has focused on older adults. When compared to other studies, the GPS for participants in this study are less than Devetak et al. [19] with $13.9(\mathrm{SD}=2.4)$, but greater than Fukuchi et al. [22] $8.0(\mathrm{SD}=3.1)$. A lower GPS reported by Fukuchi et al. [22] suggests that the quality of walking by participants in that study is better than ours and Devetak et al. [19] but this is likely due to the level of neurological impairment. Nor this study or those studies have accounted for the level of neurological impairment (defined by CT scan or MRI) when interpreting the GPS and is a limitation of our and those studies.

The clinical implications of this research will be to provide results to an already limited evidence base on how a stroke affects the quality of gait, but in particular it is the first study to report this in young adults who have had a stroke. The incidence of stroke in young adults has increased by $40 \%$ in the past decade [25], but there is little research targeted to the young population, therefore our work is key to bettering an understanding of gait in stroke, and designing rehabilitation programmes to improve walking performance in young stroke.

The narrow 95\% confidence intervals for the GPS indicate marginal inter-participant variation, but other studies have suggested a wide range in temporal, spatial, joint kinematic and kinetic parameters $[1,5$, $8,11,12,26]$. This may well be due to the number of parameters used in the GPS $(n=15)$ which could mean that high gait variables score for some joints or some participants are cancelled out by low scores in others when calculating the GPS. Therefore, the GPS might not reflect the range in the quality of walking post-stroke if there is a high inter-participant variation within a sample which there often is when measuring gait in stroke survivors. This is potentially a limitation of the GPS, but more likely reflective of the variability within our sample with regards to variation in age, time since stroke, level of disability, type of, location and cause of stroke which all affect gait post-stroke. Although our participant cohort is larger than other studies reporting the GPS in stroke patients $[19,22]$ and other conditions $[21,27]$ it is still relatively small when compared to studies reporting this data in other patient groups [13].

Other limitations of our study include the small sample size of the able-bodied control group $(n=15)$, in the stroke group the ratio between male and female participants was uneven with $\mathrm{n}=32$ (male) and $\mathrm{n}=7$ (female) while for the control it was relatively even at $\mathrm{n}=7$ (male) and $\mathrm{n}=8$ (female). Other studies [28] have reported differences in joint kinematics between males and females so this may have caused some differences between our stroke group and control. GPS uses lower limb data only and therefore whilst it provides a measure of lower limb quality of movement during walking, it does not include the effect of the movement of the torso, head and arms on lower limb movement. Although we captured which side was paretic or non-paretic, we did not capture which was the dominant side as this may also affect walking performance if the dominant side is affected following a stroke compared to non-dominant side.

\section{Conclusion}

The GPS is higher in young stroke survivors compared to the ablebodied control. This study reports that the GPS is strongly related to the efficiency of walking, temporal, and spatial parameters. This suggests that it is not only the magnitude of joint movement that is affected post-stroke, but also the quality of that movement which may help to inform rehabilitation for stroke survivors.

\section{Source of funding}

Stroke Research, Innovation and Education Fund, Wales, United Kingdom 2018-2019.

\section{Conflict of interest}

None.

\section{Acknowledgements}

Technical assistance from Mr Anthony Gregory and Mr Mark Binks from Manchester Metropolitan University is acknowledged.

\section{Appendix A. Supporting information}

Supplementary data associated with this article can be found in the online version at doi:10.1016/j.gaitpost.2021.10.037.

\section{References}

[1] H.L. Jarvis, S.J. Brown, M. Price, C. Butterworth, R. Groenevelt, K. Jackson, L. Walker, N. Rees, A. Clayton, N.D. Reeves, Return to employment after stroke in young adults: how important is the speed and energy cost of walking? Stroke 50 (11) (2019) 3198-3204, 3198-204.

[2] L.N. Awad, J.A. Palmer, R.T. Pohlig, S.A. Binder-Macleod, D.S. Reisman, Walking speed and step length asymmetry modify the energy cost of walking after stroke, Neurorehabil. Neural Repair 29 (5) (2015) 416-423.

[3] B. Brouwer, K. Parvataneni, S.J. Olney, A comparison of gait biomechanics and metabolic requirements of overground and treadmill walking in people with stroke, Clin. Biomech. 24 (9) (2009) 729-734.

[4] M.D. Lewek, E.P. Randall, Reliability of spatiotemporal asymmetry during overground walking for individuals following chronic stroke, J. Neurol. Phys. Ther. 35 (3) (2011) 116-121.

[5] S. Nadeau, M. Betschart, F. Bethoux, Gait analysis for poststroke rehabilitation: the relevance of biomechanical analysis and the impact of gait speed, Phys. Med. Rehabil. Clin. N. Am. 24 (2) (2013) 265-276.

[6] K.K. Patterson, W.H. Gage, D. Brooks, S.E. Black, W.E. McIlroy, Evaluation of gait symmetry after stroke: a comparison of current methods and recommendations for standardization, Gait Posture 31 (2) (2010) 241-246.

[7] M.M. Platts, D. Rafferty, L. Paul, Metabolic cost of over ground gait in younger stroke patients and healthy controls, Med. Sci. Sports Exerc. 38 (6) (2006) 1041-1046. 
[8] S.A. Roelker, M.G. Bowden, S.A. Kautz, R.R. Neptune, Paretic propulsion as a measure of walking performance and functional motor recovery post-stroke: a review, Gait Posture 68 (2019) 6-14.

[9] J. Boudarham, N. Roche, D. Pradon, C. Bonnyaud, D. Bensmail, R. Zory, Variations in kinematics during clinical gait analysis in stroke patients, PLoS One 8 (6) (2013), e66421.

[10] A.A. Carmo, A.F. Kleiner, P.H. Costa, R.M. Barros, Three-dimensional kinematic analysis of upper and lower limb motion during gait of post-stroke patients, Braz. J Med. Biol. Res. 45 (6) (2012) 537-545.

[11] G. Chen, C. Patten, D.H. Kothari, F.E. Zajac, Gait deviations associated with poststroke hemiparesis: improvement during treadmill walking using weight support speed, support stiffness, and handrail hold, Gait Posture 22 (1) (2005) 57-62.

[12] E.C. Wonsetler, M.G. Bowden, A systematic review of mechanisms of gait speed change post-stroke. Part 1: spatiotemporal parameters and asymmetry ratios, Top Stroke Rehabil. 24 (6) (2017) 435-446.

[13] R. Baker, J.L. McGinley, M.H. Schwartz, S. Beynon, A. Rozumalski, H.K. Graham, $\mathrm{O}$. Tirosh, The gait profile score and movement analysis profile, Gait Posture 30 (3) (2009) 265-269.

[14] M.G. Benedetti, F. Catani, A. Leardini, E. Pignotti, S. Giannini, Data management in gait analysis for clinical applications, Clin. Biomech. 13 (3) (1998) 204-215, 204 15.

[15] M.H. Schwartz, A. Rozumalski, The Gait Deviation Index: a new comprehensive index of gait pathology, Gait Posture 28 (3) (2008) 351-357.

[16] L.M. Schutte, U. Narayanan, J.L. Stout, P. Selber, J.R. Gage, M.H. Schwartz, An index for quantifying deviations from normal gait, Gait Posture 11 (1) (2000) $25-31$.

[17] R. Baker, J.L. McGinley, M. Schwartz, P. Thomason, J. Rodda, H.K. Graham, The minimal clinically important difference for the Gait Profile Score, Gait Posture 35 (4) (2012) 612-615.

[18] S. Beynon, J.L. McGinley, F. Dobson, R. Baker, Correlations of the Gait Profile Score and the Movement Analysis Profile relative to clinical judgments, Gait Posture 32 (1) (2010) 129-132.
[19] G.F. Devetak, S.K. Martello, J.C. de Almeida, K.P. Correa, D.D. Iucksch, E. F. Manffra, Reliability and minimum detectable change of the gait profile score for post-stroke patients, Gait Posture 49 (2016) 382-387.

[20] M. Bigoni, V. Cimolin, L. Vismara, A.G. Tarantino, D. Clerici, S. Baudo, M. Galli, A. Mauro, Relationship between gait profile score and clinical assessments of gait in post-stroke patients, J. Rehabil. Med. 53 (5) (2021), jrm00192.

[21] C. Celletti, M. Galli, V. Cimolin, M. Castori, N. Tenore, G. Albertini, F. Camerota, Use of the gait profile score for the evaluation of patients with joint hypermobility syndrome/Ehlers-Danlos syndrome hypermobility type, Res. Dev. Disabil. 34 (11) (2013) 4280-4285.

[22] C.A. Fukuchi, M. Duarte, Gait Profile Score in able-bodied and post-stroke individuals adjusted for the effect of gait speed, Gait Posture 69 (2019) 40-45.

[23] H.L. Jarvis, N.D. Reeves, M. Twiste, R.D. Phillip, J. Etherington, A.N. Bennett, Can high-functioning amputees with state-of-the-art prosthetics walk normally? A kinematic and dynamic study of 40 individuals, Ann. Phys. Rehabil. Med. 64 (2020), 101395.

[24] E.C. Wonsetler, M.G. Bowden, A systematic review of mechanisms of gait speed change post-stroke. Part 2: exercise capacity, muscle activation, kinetics, and kinematics, Top Stroke Rehabil. 24 (5) (2017) 394-403.

[25] M.S. Ekker, E.M. Boot, A.B. Singhal, K.S. Tan, S. Debette, A.M. Tuladhar, F.E. de Leeuw, Epidemiology, aetiology, and management of ischaemic stroke in young adults, Lancet Neurol. 17 (9) (2018) 790-801.

[26] S. Kramer, L. Johnson, J. Bernhardt, T. Cumming, Energy expenditure and cost during walking after stroke: a systematic review, Arch. Phys. Med. Rehabil. 97 (4) (2016) 619-632, e1.

[27] D.S. Speciali, E.M. Oliveira, J.R. Cardoso, J.C. Correa, R. Baker, P.R. Lucareli, Gait profile score and movement analysis profile in patients with Parkinson's disease during concurrent cognitive load, Braz. J. Phys. Ther. 18 (4) (2014) 315-322.

[28] J.H. Hollman, E.M. McDade, R.C. Petersen, Normative spatiotemporal gait parameters in older adults, Gait Posture 34 (1) (2011) 111-118. 